\section{Dangers of including outcome at baseline as a covariate in latent change score models}

\author{
Kimmo Sorjonen ${ }^{1}$, Gustav Nilsonne ${ }^{1,2}$, \\ \& Bo Melin ${ }^{1}$
}

\author{
${ }^{1}$ Department of Clinical Neuroscience, Karolinska \\ Institutet, Stockholm, Sweden \\ 2 Department of Psychology, Stockholm University, \\ Stockholm, Sweden
}

\begin{abstract}
Latent change score modelling is a version of structural equation modelling for measuring change between measurements. It seems quite common to regress change on the initial value included in the calculation of the change score (i.e. $\Delta \mathrm{Y}\left(=\mathrm{Y}_{2}-\mathrm{Y}_{1}\right)$ is regressed on $\mathrm{Y}_{1}$ ). However, similarly as in simpler regression analyses, this procedure may make findings susceptible to the influence of regression to the mean. This suspicion was verified in the present simulations. An empirical application, including reanalyses of previously published data, indicated that previously claimed reciprocal promoting effects of vocabulary and matrix reasoning on each other's longitudinal development may actually be due to regression to the mean. Researchers are recommended not to regress change on the initial value included in the calculation of the change score when employing latent change score modelling, or at least to verify findings with analyses omitting this parameter.
\end{abstract}

Keywords: adjustment for baseline, latent change score modelling, matrix reasoning, re-analyses, regression to the mean, vocabulary

\section{Introduction}

Regression to the mean is a phenomenon where measures tend to change toward the mean [1]. If a person receives an unexpectedly high (low) score, we should expect a lower (higher) score on a subsequent occasion. What can be considered an unexpectedly high or low score differs between individuals. If a professional and a beginner dart player both receive a score of 15 (let us assume that the maximum score is $3 \times 10=30$ ), we should expect a larger improvement to the next round for the professional than for the beginner. And in the other direction, if they receive a score of 27 , we can expect a larger deterioration to the next round for the beginner than for the professional. Regression to the mean also functions backward in time. The beginner probably improved more from an earlier round to the round with 27 points while the professional probably deteriorated more from an earlier round to the round with 15 points. It is important to note that these predictions based on regression to the mean apply even without any change in the individuals' true dart ability between the rounds.
To include a variable as a covariate in analyses is the statistical equivalent to assuming that entities/individuals have the same value on this variable. Consequently, if we include the dart score on a certain round as a covariate in analyses, we can expect, due to regression to the mean, a positive association between professionalism (vs. amateurism) and change in the score to a subsequent round. As subsequent value is fully defined by initial value and change (subsequent value $=$ initial value + change), given that the score on a certain round is included as a covariate in the model, the association between professionalism and the score on a subsequent round will be exactly the same as the association between professionalism and change in the score from the initial to the subsequent round. In general terms, the coefficient $\beta_{1}$ will be exactly the same if predicting $E\left|\mathrm{Y}_{2}\right|=\beta_{0}+\beta_{1} \mathrm{X}+\beta_{2} \mathrm{Y}_{1}$ as when predicting $E\left|Y_{2}-Y_{1}\right|=\beta_{0}+\beta_{1} X+\beta_{2} Y_{1}$ [2]. Therefore, the predictions, based on regression to the mean and independent of any true change in dart ability, can be rephrased: If including the dart score on a certain round as a covariate, we can expect a positive association between professionalism and the score on a subsequent, or on an earlier, round.

If not including initial dart score as a covariate in the analyses, the predictions based on regression to the mean no longer apply. As the expected differences in dart score between professionals and amateurs on two randomly selected rounds are the same, E|score $1_{\text {pro }}$ - score $1_{\text {ama }}|=E|$ score $2_{\text {pro }}-$ score $2_{\text {ama }}|=E|$ score $_{\text {pro }}|-E|$ score $_{\text {ama }} \mid$, the expected change in score between two randomly selected rounds is the same for professionals and for amateurs, E|score $2_{\text {pro }}-$ score $_{\text {pro }}|=E|$ score $2_{\text {ama }}-$ score $1_{\text {ama }}=0$. Consequently, if not including the score on a certain round as a covariate, regression to the mean does not predict any association between professionalism and change in the score from a certain round to a subsequent round. Therefore, compared with adjusted effects, unadjusted effects of a predictor, e.g. professionalism, on a change score on the dependent variable, e.g. dart ability measured as dart score, tend to be less affected by regression to the mean and give less biased estimates of the true association between the predictor and change on the dependent variable, at least if there is, as in our example, an association between the predictor and the general level of the dependent variable and if the dependent variable is measured with error [3-6].

Latent change score modeling is a form of structural equation modeling for analyzing change between measurements [7-9]. A subsequently measured value on the outcome variable, $\mathrm{Y}_{2}$, is regressed on the initial value, $\mathrm{Y}_{1}$, and a latent change factor, $\Delta Y$, where both of the regression weights are fixed to 1 , i.e. we define that $\mathrm{Y}_{2}=\mathrm{Y}_{1}+\Delta \mathrm{Y}$ and, consequently, that $\Delta \mathrm{Y}=\mathrm{Y}_{2}-\mathrm{Y}_{1}$ (Figure 1, Panel A). Other key features are that while the variance in $\mathrm{Y}_{1}$ 
and $\Delta \mathrm{Y}$ are usually freely estimated $\left(\sigma^{2} \mathrm{Y} 1\right.$ and $\sigma^{2}{ }_{\Delta \mathrm{Y}}$, respectively), the (error) variance in $\mathrm{Y}_{2}$ is set to zero, i.e. $\mathrm{Y}_{2}$ is fully defined by $\mathrm{Y}_{1}$ and $\Delta \mathrm{Y}$. Similarly, the intercept (the triangle in the figure), corresponding to the mean, of $\mathrm{Y}_{1}$ and $\Delta \mathrm{Y} \quad\left(\mu_{\mathrm{Y} 1}\right.$ and $\mu_{\Delta \mathrm{Y}}$, respectively) are freely estimated while it is set to zero for $\mathrm{Y}_{2}$, meaning, in accordance with its definition, that $\mathrm{Y}_{2}$ is assumed to be zero if both $\mathrm{Y}_{1}$ and $\Delta \mathrm{Y}$ are zero. Furthermore, although not an essential part of the latent change score model, it seems quite common to regress the latent change score, $\Delta \mathrm{Y}$, on the initial value on the outcome, $\mathrm{Y}_{1}$ $\left(\beta_{\mathrm{Y} 1, \Delta \mathrm{Y}}\right)$. The model can be extended by regressing $\Delta \mathrm{Y}$ on some predictor $\mathrm{X}\left(\beta_{\mathrm{X}, \Delta \mathrm{Y}}\right)$ in order to evaluate if $\mathrm{X}$ can predict change in $\mathrm{Y}$ between the measurements.

If the outcome variable, $\mathrm{Y}$, is measured with several indicators, $\mathrm{Y}_{1}$ and $\mathrm{Y}_{2}$ can be defined as latent variables (Figure 1, Panel B) [7, 9]. In this case we wish to achieve measurement invariance, i.e. that a possible difference between $\mathrm{Y}_{1}$ and $\mathrm{Y}_{2}$ and, consequently, a value on $\Delta \mathrm{Y}$ that differs from zero, indicates a difference in degree and not that $\mathrm{Y}_{1}$ and $\mathrm{Y}_{2}$ measure different constructs $[10,11]$. To impose measurement invariance we can (1) fix the factor loadings of the same item to be equal at different measurements, e.g. the factor loading of $\mathrm{Y}_{1.1}$ and $\mathrm{Y}_{2.1}, \lambda_{1}$, are fixed to equality; (2) fix unexplained residual variance of the same item to equality across measurements, e.g. the residual variance of $\mathrm{Y}_{1.1}$ and $\mathrm{Y}_{2.1}, \varepsilon_{1}$; (3) fix the intercept of the same item to equality across measurements, e.g. the intercept of $\mathrm{Y}_{1.1}$ and $\mathrm{Y}_{2.1}$ (not shown in the figure) [7, 11]. Additionally, it is common to allow correlations between the error terms of the same item across measurements in order to account for item-specific residuals [7, 12].
The use of latent change score models, rather than simpler regression models, has been recommended [7]. However, latent change scores in single item models (see Figure 1, panel A) are not free of measurement error [6]. Consequently, we suspected that similarly as simpler models, latent change score models may also be susceptible to the influence of regression to the mean and indicate an effect of a predictor, $\mathrm{X}$, on the latent change score, $\Delta \mathrm{Y}$, even when no true change in the outcome, $\mathrm{Y}$, has taken place. We suspected that such spurious effects may occur if conditioning the change score, $\Delta \mathrm{Y}$, on the initial value on the outcome variable, $\mathrm{Y}_{1}$, at least if there is an association between the predictor, $\mathrm{X}$, and the true value on $\mathrm{Y}$ and if $\mathrm{Y}$ is measured with error. The objective of the present simulation study was to evaluate the correctness of this suspicion, both in single item and multiple item models. A further objective was to illustrate possible problems with including the initial value on the outcome as a covariate by re-analyzing empirical data used by Kievit et al. [13].

\section{Methods}

\section{Simulation}

Virtual participants $(N=10,000)$ were allocated an $\mathrm{X}$-score from a random normal distribution (Figure 2). Then they were allocated a $\mathrm{Y}_{\text {true-score }}$ from a random normal distribution with a defined population correlation with the $\mathrm{X}$-score, $\beta_{\mathrm{X}, \mathrm{Ytrue}}$. The correlation was set to all values between 0 and 1 in steps of 0.001 , i.e. one thousand and one different values were used. In the next step, participants were allocated $\mathrm{Y}_{1}$ and $\mathrm{Y}_{2}$ scores, corresponding to observed values on the outcome at two different occasions. The population correlation between $\mathrm{Y}_{\text {true }}$

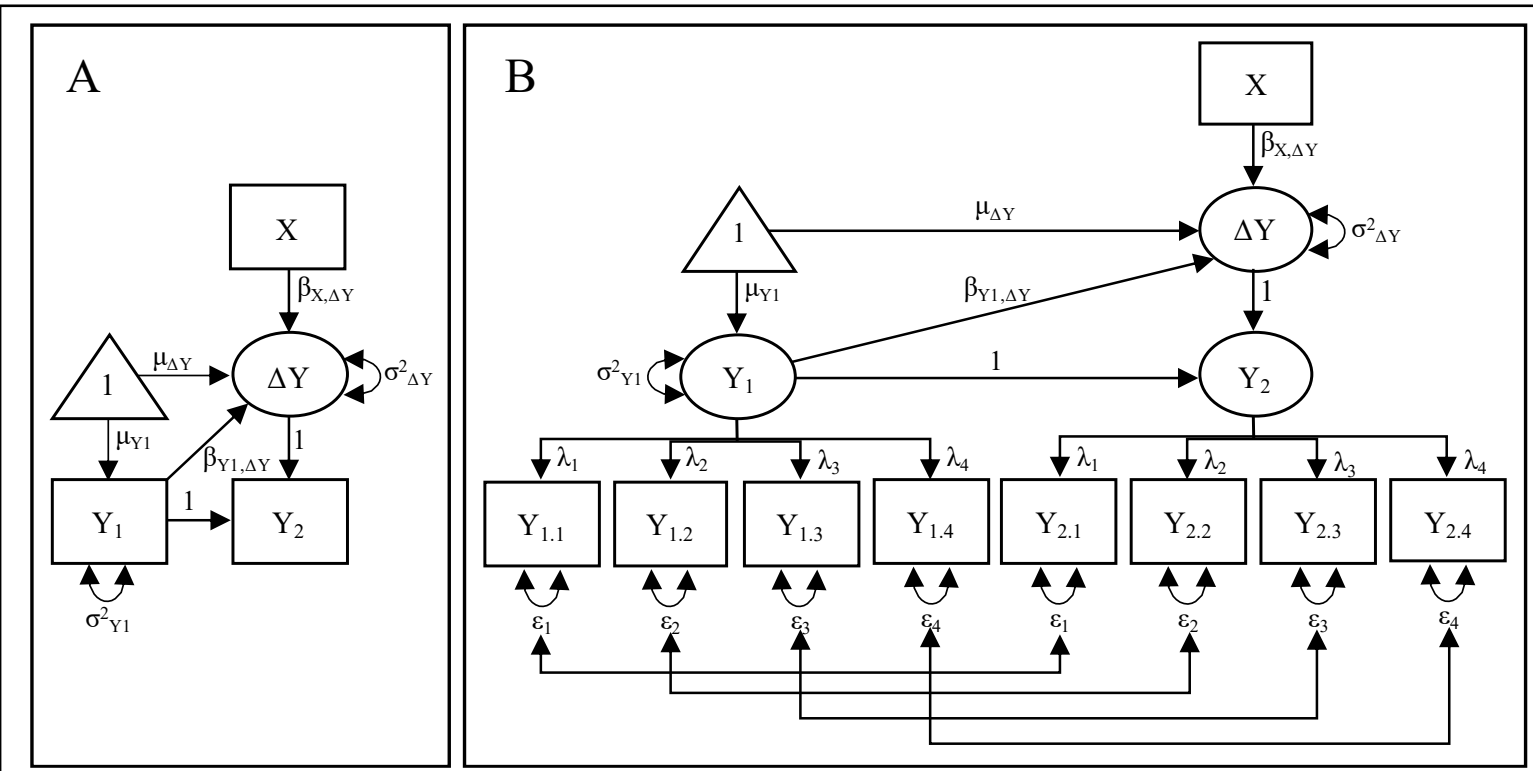

Figure 1. Illustration of latent change score modelling when the outcome, $\mathrm{Y}$, is measured with a single item on two occasions (A) and when the outcome is measured with multiple items on two occasions (B). See the text for descriptions of the parameters. 
and $\mathrm{Y}_{1} / \mathrm{Y}_{2}, \beta_{\mathrm{Y}}$, was set to either $0.5,0.7$, or 0.9 (same for both), corresponding to the square root of the test-retest reliability in the measurement of the outcome. In the simulations with multiple indicators (Figure 2, panel B), in an additional step, participants were allocated scores on four indicators of $Y_{1}$ and four indicators of $Y_{2}$, respectively. The population correlation between $\mathrm{Y}_{1}$ and $\mathrm{Y}_{2}$ and their indicators, $\beta_{\mathrm{Y}}$, was set to the same value, i.e. $0.5,0.7$, or 0.9 , as the correlation between $Y_{\text {true }}$ and $Y_{1}$ and $\mathrm{Y}_{2}$.

\section{Empirical application}

We re-analyzed data used by Kievit et al. [13], who collected data on matrix reasoning and vocabulary from 563 adolescents and young adults on two occasions, on average 1.48 years apart (an additional 221 individuals were measured at the first occasion). Measures were conducted with Wechsler Abbreviated Scale of Intelligence (WASI-II). Kievit et al. [13] used latent change score modeling and found that high initial vocabulary predicted a larger increase in the matrix reasoning score between the occasions and that a high initial matrix reasoning score predicted a larger increase in vocabulary. They concluded that their finding supported the mutualism theory of cognitive development, according to which basic cognitive abilities reciprocally facilitate (causally) each other's longitudinal development [14]. Kievit et al. [13] have made their data and scripts available at Open Science Framework at https://osf.io/93m2z/.

However, in their analyses Kievit et al. [13] regressed the change score on the initial value of the same ability, i.e. change in matrix reasoning score was regressed on initial matrix reasoning score in addition to initial vocabulary and change in vocabulary was regressed on initial vocabulary in addition to initial matrix reasoning score. Given the positive association between matrix reasoning scores and vocabulary, we suspect that this procedure may have made the findings by Kievit et al. [13] susceptible to regression to the mean.

\section{Statistical analyses}

Data, both simulated and empirical, were analyzed with latent change score models where the latent change score, $\Delta \mathrm{Y}$, was regressed on a predictor, $\mathrm{X}$, as described above and illustrated in Figure 1 (further illustrations in the Result section). The main manipulation was to either regress the latent change score on the initial value on the outcome variable, i.e. including the parameter $\beta_{\mathrm{Y} 1, \Delta \mathrm{Y}}$ in the model (Figure 1), or not. We also conducted analyses where $Y_{1}$ and $Y_{2}$ swopped positions in the models (see Figure 1 and the Result section). In this case, the latent change score corresponds to change backward in time, i.e. how much the outcome, Y, has decreased from the first to the second measurement. The effect of the predictor, $\mathrm{X}$, on this backward change should have an opposite sign compared with the effect on forward change, otherwise the effect seems to be due to regression to the mean. Simulations and analyses were conducted with $\mathrm{R}$ 4.1.0 statistical software [15] employing the lavaan package [16]. Scripts, which also generate the simulated data, are available at Open Science Framework at https://osf.io/pw8ym/.

\section{Results}

\section{Simulation}

When data were generated as in Figure 2 there was no true change in the outcome, $\mathrm{Y}$, and a significant effect of $\mathrm{X}$ on the latent change in $\mathrm{Y}$, $\beta_{\mathrm{X}, \Delta \mathrm{Y}}$, should be viewed as a type 1 error. It is apparent in Figure 3 that the likelihood for type 1 errors was high, especially with a strong association between $X$ and $Y_{\text {true }}$ (high value on the $\mathrm{X}$-axis) and when the effect of $\mathrm{X}$ on the latent change score, $\Delta \mathrm{Y}$,

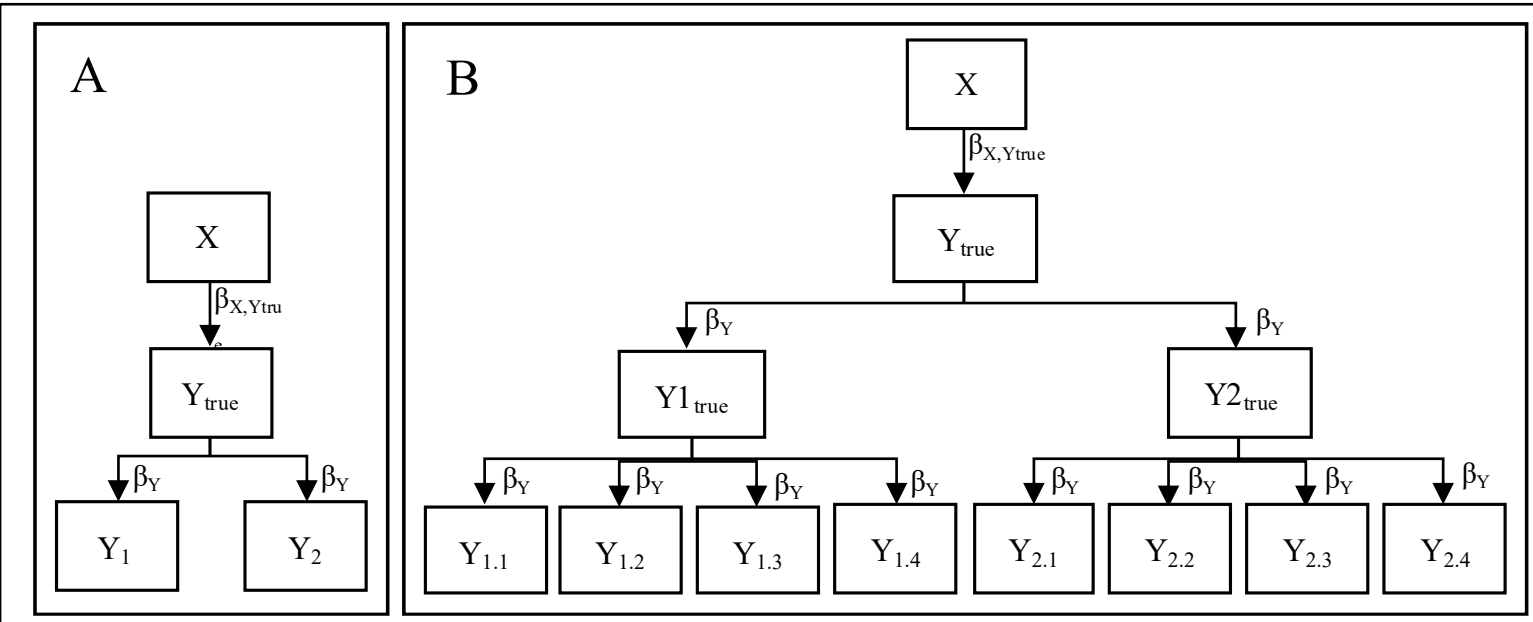

Figure 2. Illustration of data generation with no true change in the outcome, $\mathrm{Y}$, between measurements, when the outcome is measured with a single item (A) and multiple items (B) on each occasion. Y1 $1_{\text {true }}$ and $\mathrm{Y} 2_{\text {true }}$ in panel B correspond to $Y_{1}$ and $Y_{2}$ in panel $A$, respectively. See the text for descriptions of the parameters 
was calculated while adjusting for the initial value on the outcome, $\mathrm{Y}_{1}$ (lines with squares as markers). When adjusting for the initial value on the outcome, the risk for type 1 errors was not alleviated by a strong association between $\mathrm{Y}_{\text {true }}$ and $\mathrm{Y}_{1} / \mathrm{Y}_{2}$ (and between $Y_{1}$ and $Y_{2}$ and their indicators in the simulations with multiple items, second row in Figure 3), i.e. high reliability in the measurement of the outcome (the lines with squares as markers remain high in panels $\mathrm{C}$ and $\mathrm{F}$ ). It is interesting to note that the effect of $\mathrm{X}$ on $\Delta \mathrm{Y}$ was the same when $\Delta \mathrm{Y}$ represented increase in $\mathrm{Y}$ from the initial to the subsequent measurement (gray lines) and when it represented increase in $\mathrm{Y}$ backward in time from the subsequent to the initial measurement, i.e. decrease from the first to the second measurement (black lines).

The likelihood for type 1 errors was much lower when not adjusting the effect of $\mathrm{X}$ on $\Delta \mathrm{Y}$ for the initial value on the outcome (the lines with triangles as markers in Figure 3), especially in the models with a single item (panels $\mathrm{A}, \mathrm{B}$, and $\mathrm{C}$, data generated as in panel $A$ in Figure 2). When analyzing data with multiple items (panels D, E, and $\mathrm{F}$, data generated as in panel $\mathrm{B}$ in Figure 2), there was an increase in the likelihood for type 1 errors also when not adjusting for the initial value on the outcome, especially if the outcome was measured with low reliability (panel D). When adjusting for the initial value on the outcome, the likelihood for type 1 errors was the same when analyses were conducted with a single item and a multiple items model (the lines with squares as markers are identical in panels $\mathrm{A}-\mathrm{C}$ and in panels $\mathrm{D}-\mathrm{F}$, respectively).

\section{Empirical application}

Re-analyses of data from Kievit et al. [13] indicated, as in the original study, that when including initial value on the outcome ability as a covariate, a high initial vocabulary predicted a larger increase in the matrix reasoning score between measurement occasions and a high initial matrix reasoning score predicted a larger increase in vocabulary (Figure 4, panels A and B). However, when predicting change backward in time and conditioning on the subsequent value on the outcome ability, the effects remained positive and of a similar strength (Figure 4, panels C and D). This means that here, with the same data, a high initial vocabulary predicted a smaller increase in the matrix reasoning score and a high initial matrix reasoning score predicted a smaller increase in vocabulary. When change was not conditioned on the initial value on the outcome ability, initial vocabulary and initial matrix reasoning score had an effect on each other but hardly any effect on change in the other ability (Figure 4, panels E and F).

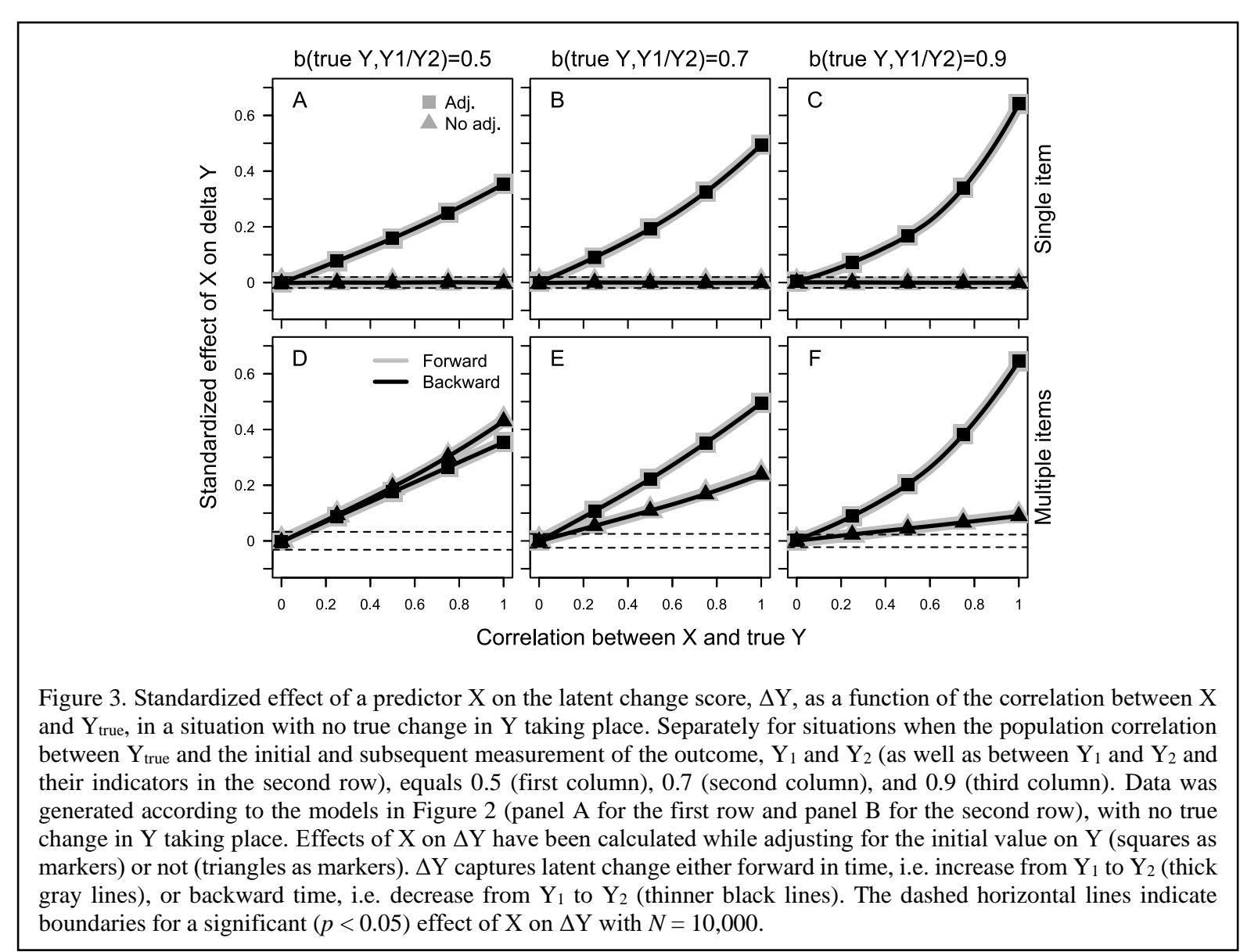




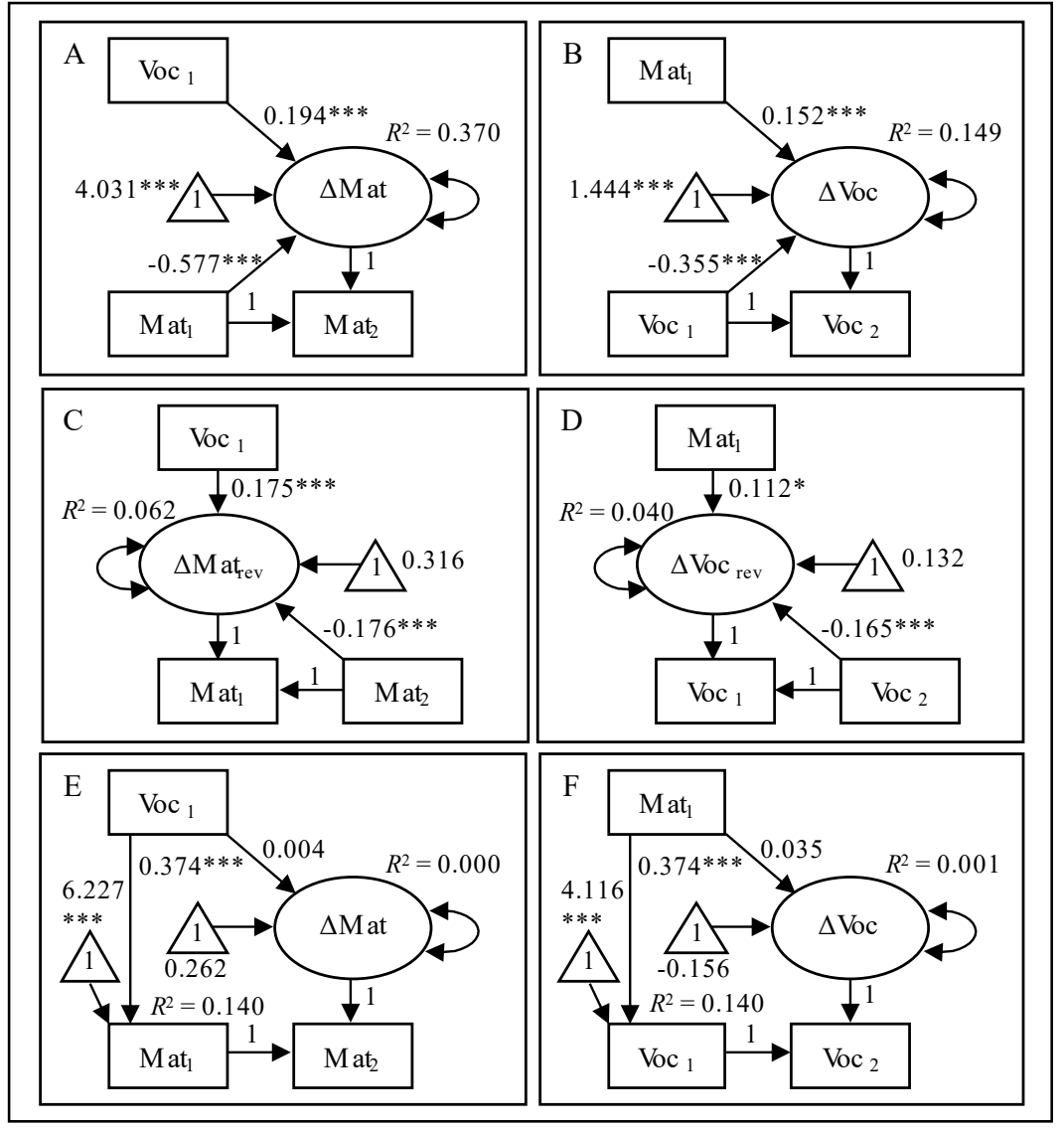

The effects in Figure 4 are illustrated in Figure 5. When conditioning change on initial value on the outcome ability (in this example the mean), those with high (one $S D$ above the mean) initial vocabulary were predicted to have increased more in the matrix reasoning score between measurements compared with those with average and low (one $S D$ below the mean) initial vocabulary (gray lines in panel A in Figure 5). Similarly, the vocabulary of those with a high initial matrix reasoning score was predicted to have increased more between occasions compared with those with a lower initial matrix reasoning score (gray lines in panel $\mathrm{C}$ in Figure 5). However, if predicting change backward in time and conditioning on the subsequent value on the outcome, those with high initial vocabulary (matrix reasoning score) were predicted to have had a higher initial matrix reasoning score (vocabulary) and, consequently, to have increased less between occasions compared with those with a lower initial vocabulary (matrix reasoning score) (black lines in panels A and C in Figure 5).

When not including initial value on the outcome ability as a covariate, but instead regressing the initial abilities on each other (as in panels $\mathrm{E}$ and $\mathrm{F}$ in Figure 4), high initial vocabulary (matrix reasoning score) predicted a high initial matrix reasoning score (vocabulary) but not change in matrix reasoning score (vocabulary) between occasions, which was predicted to be slightly positive irrespective of initial vocabulary (matrix reasoning score) (gray lines in panels B and D in Figure 5). This corresponded quite
Figure 4. Latent change score models with change in matrix reasoning score predicted from initial vocabulary (panels $\mathrm{A}, \mathrm{C}$, and $\mathrm{E}$ ) and change in vocabulary predicted from initial matrix reasoning score (panels $\mathrm{B}, \mathrm{D}$, and F) when including the initial value on the outcome ability as a covariate (panels A and B), when predicting change backward in time and including the subsequent value on the outcome ability as a covariate (panels C and D), and when not including initial value on the outcome as a covariate (panels E and F). Standardized coefficients. $* p<.05$, *** $p<$ .001 .

well with actual mean matrix reasoning scores for those with high (upper third), average (middle third), and low (bottom third) initial vocabulary (dotted black lines in panel B in Figure 5) and actual mean vocabulary for those with high, average, and low initial matrix reasoning score (dotted black lines in panel D in Figure 5).

\section{Discussion}

The present simulations showed that if regressing change on the initial value on the outcome variable, results from latent change score modelling are susceptible to regression to the mean. With an association between a predictor, $\mathrm{X}$, and the true value on the outcome variable, $\mathrm{Y}$, findings can indicate that $\mathrm{X}$ has an association with change in $\mathrm{Y}$ even if no true change has taken place. This is in accordance with a similar effect demonstrated in simpler regression models [3-6]. Additionally, the present simulations indicated that this problem might not be alleviated by high reliability in the measurement of the outcome or by measuring the outcome with several indicators and defining the outcome as a latent, rather than as an observed, variable.

In single item models, the risk for a spurious effect, due to regression to the mean, of $\mathrm{X}$ on the change in $\mathrm{Y}$ was no longer present if removing the effect of initial $\mathrm{Y}$ on the change in $\mathrm{Y}$ from the model. However, in multiple item models, where $Y$ at the two occasions was defined as a latent variable, the risk for spurious findings was present also when not regressing change in $\mathrm{Y}$ on initial $\mathrm{Y}$, especially if $\mathrm{Y}$ was measured with low reliability. This may indicate some kind of negative consequence of high power in combination with bias, where the multiple item model somehow manages to identify a signal among all the noise even when it should not. 


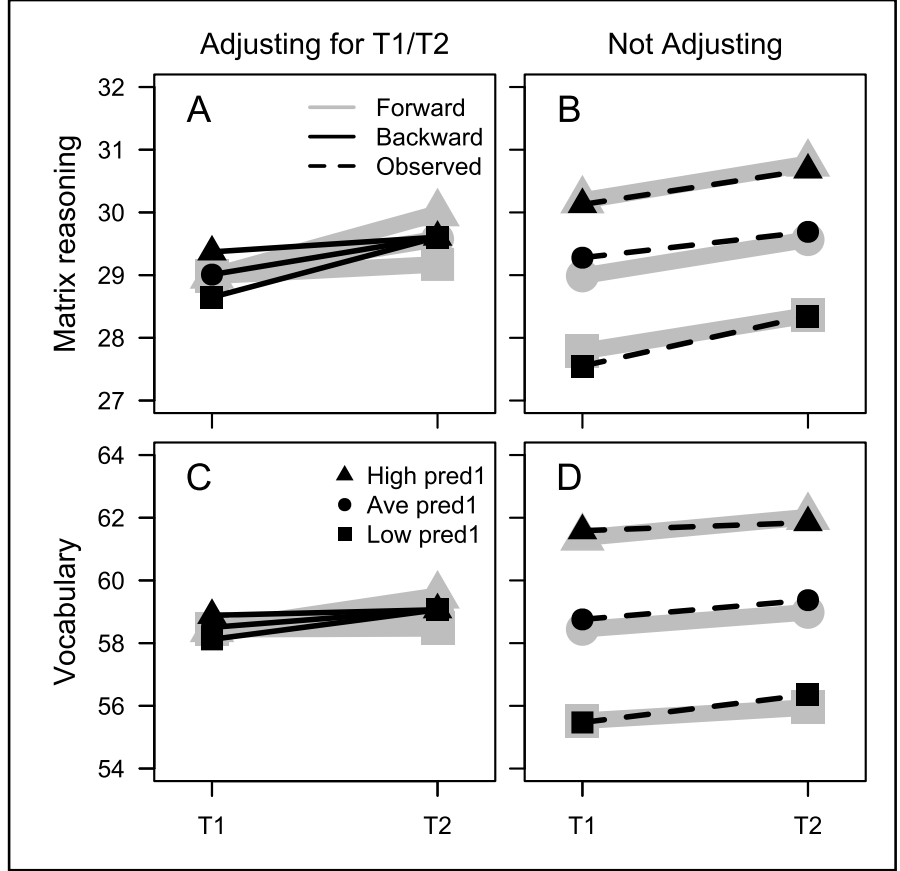

In the empirical re-analyses, if including the initial value on the outcome variable as a covariate in the model, initial vocabulary and initial matrix reasoning had positive effects on change in matrix reasoning and on change in vocabulary between two measurement occasions, respectively. This is, not surprisingly, in line with the findings in the original study by Kievit et. al [13]. These authors concluded that the results supported the mutualism theory of cognitive development, according to which cognitive abilities promote the development of other cognitive abilities [14]. However, if reversing the latent change score factor, so that it captured change backward in time from the subsequent to the initial occasion, and conditioning change on the subsequent value on the outcome variable, the effects of initial vocabulary and initial matrix reasoning score on the change in matrix reasoning and vocabulary, respectively, remained positive. This would mean that a high score on one ability predicts a smaller increase on the other ability.

Surely, if analyses of the same data can be used to conclude both $\mathrm{X}$ and its exact opposite, the validity of either conclusion can be doubted. We propose that a more tenable description of the findings is that if conditioning on measured vocabulary (matrix reasoning) at a certain occasion we can, due to regression to the mean, expect a positive association between matrix reasoning score (vocabulary) and another measured value, either forward or backward in time, on vocabulary (matrix reasoning). The conclusion that observed adjusted associations probably are due to regression to the mean, rather than any true associations between initial/subsequent ability and change in the other ability, were supported by the fact that the association between initial vocabulary (matrix reasoning) and change in matrix reasoning
Figure 5. Predicted change in matrix reasoning (panels A and B) and vocabulary (panels $\mathrm{C}$ and D) from the first to the second (gray lines) and from the second to the first (solid black lines) measurement, when conditioning on the initial (for forward predictions) and the subsequent (for backward predictions) mean on the outcome ability (panels A and C) and without conditioning (panels B and D). The dashed black lines indicate the actual means. Separately for those with high (one $S D$ above the mean for predictions and the upper third for the actual means, triangles), average (middle third, circles), and low (one $S D$ below the mean / bottom third, squares) initial value on the predictor (i.e. initial vocabulary in panels $\mathrm{A}$ and $\mathrm{B}$ and initial matrix reasoning score in panels $\mathrm{C}$ and D). Note: The predictions are based on the models in Figure 4, the gray lines in the present panels $\mathrm{A}, \mathrm{C}, \mathrm{B}$, and $\mathrm{D}$ on the models in panels $\mathrm{A}$, $\mathrm{B}, \mathrm{E}$, and $\mathrm{F}$ in Figure 4, respectively, and the solid black lines in the present panels $\mathrm{A}$ and $\mathrm{C}$ on the models in panels C and D in Figure 4, respectively.

(vocabulary) vanished if not adjusting for initial matrix reasoning (vocabulary).

\section{Limitations}

In the simulations with multiple indicators, the observed scores were assumed to be indicators, measured with error, of true occasion-specific indicators, including occasion-specific error, of individuals' true general value on the outcome variable (see panel B in Figure 2). We believe this hypothetical situation to be realistic. The occasionspecific measurement errors could be due to factors such as temporary illness or stressful events (missing the bus on the way to the measurement location) or low motivation. Of course, other data generating models, for example without occasionspecific measurement errors, are possible. However, we believe it would be a severe logical slip to conclude that because other data generating models are possible, the results from the present simulations must be wrong and that results from latent change score modelling with multiple indicators can never be susceptible to the influence of regression to the mean.

The findings in the present re-analyses of empirical data should not be interpreted as a final refutation of the mutualism theory of cognitive development, but rather as a critique of the alleged evidence for the theory in Kievit et al. [13]. In a later study with three waves of data, Kievit et al. [17] employed latent growth modelling. Without adjustment for the initial value on the outcome variable, they found a positive association between predicted initial vocabulary (matrix reasoning score) and predicted individual slopes in matrix reasoning (vocabulary) across the three measurements. These 
findings should be less susceptible to the influence of regression to the mean.

\section{Conclusions}

As in simpler regression models, if including outcome at baseline as a covariate in latent change score models, the effect of a predictor, $\mathrm{X}$, on the latent change in the outcome variable, $\mathrm{Y}$, is susceptible to regression to the mean. Given an association between $\mathrm{X}$ and the true value on $\mathrm{Y}$, the analyses may indicate an effect of $\mathrm{X}$ on the change in $\mathrm{Y}$ even if no true change has taken place. Consequently, researchers are recommended not to regress change on the initial value on the outcome variable when conducting latent change score modelling, or at least to confirm their findings with analyses not including this parameter.

If they are, for some reason, unwilling to remove the effect of initial outcome on change in the outcome from their models, researchers could confirm the logic of their conclusions by reversing the latent change factor so it captures change from the subsequent to the initial measurement. If not due to regression to the mean, this reversal of change should reverse the sign of the effect of $X$ on the change in Y. Analyses of effects on reversed change are also recommended for multiple item models, where the outcome is defined as latent variables, as these models seem to suffer from an increased risk for type 1 errors even if not regressing change on initial outcome.

\section{References}

1. Galton F. Regression towards mediocrity in hereditary stature. The Journal of the Anthropological Institute of Great Britain and Ireland. 1886;15:246-63. doi:10.2307/2841583.

2. van Breukelen GJP. ANCOVA versus CHANGE from baseline in nonrandomized studies: The difference. Multivariate Behavioral Research. 2013;48:895-922. doi:10.1080/00273171.2013.831743.

3. Eriksson K, Häggström O. Lord's paradox in a continuous setting and a regression artifact in numerical cognition research. PLoS ONE. 2014;9:e95949. doi:10.1371/journal.pone.0095949.

4. Sorjonen K, Melin B, Ingre M. Predicting the effect of a predictor when controlling for baseline. Educational and Psychological Measurement. 2019;79:688-98. doi: $10.1177 / 0013164418822112$.

5. Glymour MM, Weuve J, Berkman LF, Kawachi I, Robins JM. When is baseline adjustment useful in analyses of change? An example with education and cognitive change. American Journal of Epidemiology. 2005;162:267-78. doi:10.1093/aje/kwi187.

6. Castro-Schilo L, Grimm KJ. Using residualized change versus difference scores for longitudinal research.
Journal of Social and Personal Relationships. 2018;35:32-58. doi:10.1177/0265407517718387.

7. Kievit RA, Brandmaier AM, Ziegler G, van Harmelen A-L, de Mooij SMM, Moutoussis M, et al. Developmental cognitive neuroscience using latent change score models: A tutorial and applications. Developmental Cognitive Neuroscience. 2018;33:99117. doi:10.1016/j.den.2017.11.007.

8. Ghisletta P, McArdle JJ. Latent curve models and latent change score models estimated in R. Structural Equation Modeling: A Multidisciplinary Journal. 2012;19:651-82. doi:10.1080/10705511.2012.713275.

9. McArdle JJ. Latent variable modeling of differences and changes with longitudinal data. Annu Rev Psychol. 2009;60:577-605.

doi:10.1146/annurev.psych.60.110707.163612.

10. Wicherts JM. The importance of measurement invariance in neurocognitive ability testing. The Clinical Neuropsychologist. 2016;30:1006-16. doi:10.1080/13854046.2016.1205136.

11. Widaman KF, Ferrer E, Conger RD. Factorial invariance within longitudinal structural equation models: Measuring the same construct across time. Child Development Perspectives. 2010;4:10-8. doi:10.1111/j.1750-8606.2009.00110.x.

12. Wheaton B, Muthen B, Alwin DF, Summers GF. Assessing reliability and stability in panel models. Sociological Methodology. 1977;8:84. doi:10.2307/270754

13. Kievit RA, Lindenberger U, Goodyer IM, Jones PB, Fonagy P, Bullmore ET, et al. Mutualistic coupling between vocabulary and reasoning supports cognitive development during late adolescence and early adulthood. Psychol Sci. 2017;28:1419-31. doi:10.1177/0956797617710785.

14. van der Maas HLJ, Dolan CV, Grasman RPPP, Wicherts JM, Huizenga HM, Raijmakers MEJ. A dynamical model of general intelligence: The positive manifold of intelligence by mutualism. Psychological Review. 2006;113:842-61. doi:10.1037/0033295X.113.4.842.

15. R Core Team. R: A language and environment for statistical computing. R Foundation for Statistical Computing, Vienna, Austria. URL https://www.Rproject.org/. 2021.

16. Rosseel Y. lavaan: An R package for structural equation modeling. Journal of Statistical Software. 2012;48:1-36. https://www.jstatsoft.org/v48/i02/.

17. Kievit RA, Hofman AD, Nation K. Mutualistic coupling between vocabulary and reasoning in young children: A replication and extension of the study by Kievit et al. (2017). Psychol Sci. 2019;30:1245-52. doi:10.1177/0956797619841265. 